\title{
Le Défi De La Scolarisation Primaire Universelle Des Filles Dans La Région Du Worodougou En Côte D'Ivoire
}

\author{
Yeo Soungari \\ Kei Mathias
}

IREEP, Université Félix HOUPHOUËT-BOIGNY de Cocody, Abidjan

doi: 10.19044/esj.2016.v12n25p393 URL:http://dx.doi.org/10.19044/esj.2016.v12n25p393

\begin{abstract}
Under-education and schooling are important phenomena that hinder the achievement of universal primary education, especially for girls. This article aims to analyze strategies to implement in order to achieve universal primary education for girls in the region of Worodougou, in northwestern of Côte d'Ivoire. To collect data, individual and group interviews were conducted with actors of education. After this investigation, it is concluded that the obstacles to achieving of this goal are, among others, poverty, illiteracy of the parents, social representation of women in the community, migration of girls from Worodougou region, in particular, the dream of going to settle in the west countries, and more specifically in France, etc. The proposed strategies are numerous. However, we can mention the need for the implementation of literacy programs, effectiveness of free education for all the children, increasing educational opportunities, etc.
\end{abstract}

Keywords: Challenge, under-education, schooling, girl, Worodougou

\section{Résumé}

La sous-scolarisation et la déscolarisation sont des phénomènes importants qui entravent la réalisation de l'éducation primaire universelle, notamment chez les filles. Le but de cet article est d'analyser les stratégies à mettre en œuvre en vue de la réalisation de l'éducation primaire universelle chez les filles dans la région du Worodougou, dans le nord-ouest en Côte d'Ivoire. Pour collecter les informations, des entretiens individuels et de groupe ont été réalisés avec les acteurs de l'éducation. Au terme de cette investigation, il ressort que les obstacles à la réalisation de cet objectif éducatif sont entre autres, la pauvreté, l'analphabétisme des parents, la représentation sociale de la femme dans la communauté, la migration des filles de la région du Worodougou, notamment, le rêve de partir s'établir en occident et plus 
précisément en France, etc. Les stratégies proposées sont assez nombreuses. On peut cependant évoquer la nécessité de la mise en œuvre des programmes d'alphabétisation, l'effectivité de la gratuité de l'école, l'accroissement de l'offre éducative, etc.

Mots clés: Défi, sous-scolarisation, déscolarisation, fille, Worodougou

\section{INTRODUCTION ET PROBLEMATIQUE}

L'éradication de l'analphabétisme des populations et principalement celui des filles et des nécessite qu'en amont, des stratégies efficaces soient mises en œuvre pour la réalisation de l'éducation primaire universelle, inclusive et de bonne qualité. Les différents engagements internationaux (Jomtien en 1990 ; Forum mondial de Dakar en 2000 ; Assemblée des Nations Unies en 2000 pour les OMD, etc.) n'ont pas permis d'aboutir à la réalisation de la scolarisation primaire universelle dans de nombreux pays, dont ceux de l'Afrique subsaharienne. Cependant, si on note que le nombre d'enfants scolarisés au primaire a connu une augmentation et que le pourcentage d'enfants qui n'ont jamais été scolarisés a baissé (UNESCO, 2015), force est de reconnaitre que la population non scolarisée demeure importante et que de nombreux enfants, majoritairement les filles n'achèvent pas le cycle primaire.

Ainsi, en 2012, près de 58 millions d'enfants, dont majoritairement des filles, en âge de suivre l'enseignement primaire, n'étaient pas scolarisés. Le phénomène de sous-scolarisation et même celui de la déscolarisation touchent majoritairement les filles comme le prouvent les données statistiques des différentes organisations internationales (PNUD, ONU, UNICEF). Selon le Rapport de suivi de l'EPT (Unesco, 2013), 31 millions de filles en âge de fréquenter l'école primaire n'ont pas été scolarisées et 17 millions d'entre elles n'avaient aucune chance d'aller à l'école. Ce rapport relève également des disparités de scolarisation selon le genre en défaveur des filles étant donné que le nombre de filles scolarisées dans l'enseignement primaire est inférieur de 5 millions à celui des garçons. Sur les 57 millions d'enfants non scolarisés dans le monde, 33 millions se trouvent en Afrique subsaharienne, et plus de la moitié (55\%) sont des filles (ONU, 2015).

En Côte d'Ivoire, il y a eu une progression des effectifs scolaires féminins depuis l'accession du pays à l'indépendance. Toutefois, les filles sont toujours victimes de sous-scolarisation et de la déscolarisation (Lange 1998 ; RESEN, 2015 ; PND, 2016-2020). Il existe, en matière de scolarisation primaire, de fortes inégalités entre les sexes en défaveur de la grande majorité des filles malgré l'importante hausse de la population féminine scolarisable ces dernières années. Ainsi, on note que les filles représentent $57,1 \%$ des enfants non scolarisés, et $48,7 \%$ des filles d'âge scolaire ne fréquentent pas l'école contre 35,8 \% des garçons (MEN, 2015). Les obstacles qui perpétuent 
la sous-scolarisation des filles à l'école primaire sont multiples. De nombreux auteurs (Dédy et Bih, 1997 ; Bih, 2003; Manikak, 2011; UNICEF, 2012) évoquent l'enclavement des régions et la distance du domicile par rapport à l'école, les mariages et les grossesses précoces, les représentations sociales que se font les communautés de l'école, les stéréotypes sur les rôles sexistes, l'extrême pauvreté des parents, les coûts d'écolage, le coût d'opportunité, la préférence donnée par les parents à l'apprentissage des garçon, la quantité et la qualité des équipements scolaires et la qualité des enseignants, comme des obstacles à la scolarisation des filles et leur maintien dans le cycle d'enseignement primaire. A ces obstacles, Illou (2011) ajoute des obstacles d'ordre religieux qui confèrent un autre rôle à la femme, l'analphabétisme des populations et l'inadéquation du système scolaire par rapport aux réalités locales des populations, ce système scolaire étant calqué sur celui de l'occident. Toutefois, relève le Rapport de suivi des Objectifs du Millénaire pour le Développement (2012), des obstacles individuels tels que le mauvais état de santé et la malnutrition peuvent être à l'origine de la sous-scolarisation et de la déscolarisation des enfants et principalement des filles. La pression démographique, les conflits et le manque d'engagement suffisant dans certains pays à forte population non scolarisée, le manque de capacités et la faiblesse de l'encadrement politique en matière de genre sont une contrainte à la scolarisation et à l'achèvement de la scolarité primaire des filles dans la plupart des pays d'Afrique ( Ministère de la famille, de la femme et de l'enfant, 2014 ; Unesco, 2015).

L'analyse des données statistiques et surtout des obstacles concernant la scolarisation des filles dans le monde, en Afrique et principalement en Côte d'Ivoire montre qu'il y a encore des efforts à fournir. C'est pourquoi, depuis plusieurs décennies, la question de l'éducation des filles s'est constamment posée comme une préoccupation majeure dans les assemblées de prise de décision.

D’une manière générale, il existe des disparités en matière de scolarisation primaire selon les régions en Côte d'Ivoire. Les régions du Kabadougou au nord-est, du Poro au nord, du Gbôklé au Sud-Ouest, du Gontougo au Nord Est, du Béré et du Worodougou au Nord-ouest sont reconnues comme des zones de sous scolarisation et surtout de déscolarisation de la jeune fille (MICS, 2006).Dans ce contexte, le défi de la scolarisation primaire universelle, et notamment chez les filles, suscite des interrogations au niveau des stratégies à adopter pour arriver à des résultats satisfaisants. Pour arriver à la scolarisation primaire universelle, les députés ont voté une loi portant modification de la loi $\mathrm{n}^{\circ}$ 95-696 du 7 septembre 1995 relative à l'Enseignement. Cette loi adoptée par le parlement ivoirien en septembre 2015 stipule que l'école est désormais obligatoire pour tous les enfants âgés de 6 à 16 ans. Elle oblige par conséquent tous les acteurs de l'éducation, notamment 
les parents dont les enfants appartiennent à cette tranche d'âge, à les inscrire à l'école publique ou privée et à veiller à leur assiduité sous peine de sanctions. La loi indique que l'Etat ivoirien, pour sa part, mettra progressivement à disposition les infrastructures scolaires appropriées et les personnels enseignants et d'encadrement nécessaires et qualifiés à partir de l'année scolaire 2015-2016, jusqu' à l'effectivité totale de cette mesure en 2025. Dans cette perspective, le gouvernement entend, à travers cette loi, résoudre l'épineuse question de la scolarisation primaire universelle des enfants d'âge scolaire et principalement la scolarisation primaire des filles, qui, pendant longtemps ont été victimes de la sous-scolarisation. De ce fait, il s'agit là, en ce qui concerne la scolarisation primaire universelle des filles, d'un défi à relever dans toutes les régions du pays et principalement dans les zones à faible taux de scolarisation et de maintien des filles dans le cycle d'enseignement primaire.

D’une manière générale, il existe des disparités en matière de scolarisation primaire selon les régions. Les régions du Kabadougou au nordest, du Poro au nord, du Gbôklé au Sud Ouest, du Gontougo au Nord Est, du Béré et du Worodougou au Nord-ouest sont reconnues comme des zones de sous-scolarisation et surtout de déscolarisation de la jeune fille (MICS, 2006).

Dans ce contexte, le défi de la scolarisation primaire universelle, et notamment chez les filles, suscite des interrogations au niveau des stratégies à adopter pour arriver à des résultats satisfaisants. Quelles sont les stratégies qu'il faudra-t-il mettre en œuvre pour relever le défi de la scolarisation primaire universelle chez les filles dans les zones de sous-scolarisation ou de déscolarisation en Côte d'Ivoire. Cette recherche s'intéresse au cas spécifique de la région du Worodougou dans le nord-est de la Côte d'Ivoire.

L'objectif général de cette étude est d'étudier les stratégies à mettre en œuvre pour relever le défi de la scolarisation primaire universelle des filles en Côte d'Ivoire et principalement dans la région du Worodougou.

De façon spécifique, il s'agit :

- d'identifier les obstacles liés à la scolarisation et au maintien des filles dans le cycle d'enseignement primaire ;

- d'examiner les actions menées dans la région pour résoudre le problème en question ;

- et de proposer des stratégies pour résoudre la question de la scolarisation primaire universelle chez les filles dans cette région.

\section{CADRE DE REFERENCE THEORIQUE}

Plusieurs théories peuvent apporter un éclairage à la question de la scolarisation et au maintien des filles dans le cycle d'enseignement primaire. Toutefois, nous avons recouru à la théorie des jeux pour expliquer le phénomène de sous-scolarisation et de scolarisation des filles auquel il s'agit 
d'apporter des réponses appropriées. La théorie des jeux, introduite par Von Neuman et Morgenstern, est issue du développement des mathématiques appliquées à l'économie. Elle permet d'analyser les stratégies et les comportements des joueurs ou agents économiques qui peuvent avoir des intérêts contradictoires (Silem, 2008), bien qu'une coopération dans les jeux soit envisagée entre eux.

La théorie des jeux analyse les comportements rationnels des décideurs en situation d'interaction ou d'interdépendance. Par conséquent, on estime que les résultats obtenus par un décideur, conformément à ses actions, sont fonction des actions des autres décideurs. Autrement dit, le sort de chaque participant dépend non seulement des décisions qu'il prend, mais aussi de celles prises par d'autres participants.

En ramenant cette analyse à notre étude, on pourrait dire que la réalisation de la scolarisation primaire universelle des filles dépend non seulement de l'organisation stratégique mise en place, mais aussi et surtout du comportement de toutes les personnes physiques et morales impliquées dans le domaine, de toutes structures impliquées dans le domaine y compris la population cible elle-même, c'est-à-dire les filles elles-mêmes.

L'obtention de résultats probants en la matière est fonction des différentes stratégies d'actions mises en place, de la planification des actions menées, de la coordination entre les organismes, de la disponibilité des ressources, de la prise de décisions, des stratégies de communication et d'information, du contrôle, du suivi et de l'encadrement des filles, du bon fonctionnement de chaque organisme d'enseignement primaire, de l'innovation apportée aux programmes et projets de scolarisation primaire, des parents de filles d'âge scolaire et surtout des filles qui peuvent, à leur niveau, avoir d'autres préoccupations autres que l'école. Il y a donc une interdépendance entre les différents acteurs que sont le gouvernement ivoirien qui entend promouvoir la scolarisation primaire universelle et principalement celle des filles, le ministère chargé de l'Education nationale, les structures éducatives au niveau central, les acteurs au niveau de la région (Direction Régionale de l'Education Nationale, Direction Départementale de l'Education Nationale, Inspection de l'Enseignement Préscolaire et Primaire, Conseiller Pédagogique, Conseiller pédagogique de l'Inspection, Conseiller de secteur, enseignants, les Comités de Gestion des Etablissements scolaires, Association des parents d'élèves, association des femmes, président des jeunes, les ONG, etc.), les partenaires internationaux (UNESCO, PNUD, UNICEF, etc.) et le public cible.

Dans le cadre de la réalisation de l'enseignement primaire universelle, et principalement la scolarisation et le maintien des filles dans l'enseignement primaire, chacun des acteurs ou participants prend des décisions. Toutefois, une décision prise par un participant ne peut conduire à l'obtention de résultats 
satisfaisants sans la contribution des autres acteurs ou participants. En d'autres termes, l'engagement du gouvernement à résoudre définitivement la question de la scolarisation et du maintien des filles à l'école primaire ne peut aboutir à des résultats satisfaisants si les structures éducatives centrales ou locales sont marquées par des dysfonctionnements. L'absence de volonté politique peut constituer une entrave à la réalisation du défi de la scolarisation primaire universelle, notamment chez les filles. De même, l'absence de coopération entre les acteurs cités plus haut peut justifier l'inefficacité des actions menées.

Par ailleurs, la marginalisation des communautés, des leaders communautaires, des leaders religieux, des femmes et des filles dans la prise de décisions relatives à l'éducation primaire, leur absence dans les campagnes d'information, de sensibilisation et de motivation entravent le fonctionnement normal du programme de l'Education Pour Tous et principalement la scolarisation primaire universelle des filles.

\section{METHODOLOGIE}

\subsection{Sites de l'étude}

La région du Worodougou a été choisie pour mener cette étude. Ce choix se justifie par le fait qu'il s'agit d'une région à faible taux de scolarisation primaire, et principalement une région caractérisée par la sousscolarisation et la déscolarisation des filles. L'étude s'est déroulée aussi bien en milieu urbain que rural. En zone urbaine les villes de Séguéla et de Kani ont été choisies, tandis que les villages de Téguéla et de Babien ont été choisis en zone rurale.

\subsection{Population de l'étude}

La population de l'étude est constituée de l'ensemble des acteurs concernés ou impliqués dans le domaine de l'éducation dans la région de l'étude. Il s'agit principalement :

$\checkmark$ des enseignants des écoles primaires ;

$\checkmark$ des Inspecteurs de l'Enseignement Préscolaire et Primaire ;

$\checkmark$ des Conseillers Pédagogiques de Secteur;

$\checkmark$ des Directeurs Régionaux de l'Éducation Nationale ;

$\checkmark$ des responsables de Comité de Gestion des Etablissements Scolaires (COGES) ;

$\checkmark$ des parents d'élèves

$\checkmark$ des leaders communautaires (chefs de communautés religieuses, chefs d'organisations de développement local etc.)

$\checkmark$ des filles déscolarisées;

$\checkmark$ des ONG internationales et nationales intervenant dans le domaine de l'éducation des filles. 


\subsection{Techniques de collecte des données}

Les techniques de collectes utilisées sont l'entretien individuel et le focus group.

\subsubsection{Entretiens individuels}

Les acteurs qui ont été concernés par les entretiens individuels sont :

$\checkmark$ La Direction Régionale de l'Education Nationale de Séguéla;

$\checkmark$ Les Inspections de l'Enseignement Primaire de Séguéla et de Kani

$\checkmark$ Les Conseillers pédagogiques

$\checkmark$ Les Responsables COGES des localités enquêtées;

$\checkmark$ Les leaders communautaires (chefs de villages, présidente des femmes, président des jeunes et imams)

$\checkmark$ Les responsables d'ONG dans la région

$\checkmark$ Les filles déscolarisées

Le nombre d'entretiens réalisés est consigné dans le tableau ci-dessous.

Tableau 1 : Nombre d'entretiens individuels réalisés

\begin{tabular}{|c|c|c|}
\hline Acteurs & Localités & $\begin{array}{l}\text { Nombre d'entretiens } \\
\text { individuels réalisés }\end{array}$ \\
\hline DREN & - Séguéla & 1 \\
\hline IEP de Séguéla et de Kani & $\begin{array}{l}\text { - Séguéla } \\
\text { - Kani }\end{array}$ & 2 \\
\hline Conseillers pédagogiques & $\begin{array}{l}\text {-Séguéla } \\
\text { - Kani }\end{array}$ & 2 \\
\hline Responsables COGES & $\begin{array}{l}\text { - Kani } \\
\text { - Babien } \\
\text { - Téguéla }\end{array}$ & 3 \\
\hline $\begin{array}{l}\text { Leaders communautaires (chefs de } \\
\text { villages, présidente des femmes, } \\
\text { président des jeunes et imams) }\end{array}$ & $\begin{array}{l}\text { - Kani } \\
\text { - Babien } \\
\text { - Téguéla }\end{array}$ & 12 \\
\hline Responsables d'ONG dans la région & -Séguéla & 2 \\
\hline Filles déscolarisées & $\begin{array}{l}\text { - Kani } \\
\text { - Babien } \\
\text { - Téguéla }\end{array}$ & 3 \\
\hline TOTAL DES ENTRETIENS & & 25 \\
\hline
\end{tabular}

Les entretiens avec l'ensemble des acteurs évoqués ci-dessus ont permis d'appréhender les obstacles à la scolarisation des filles dans la région, mais aussi les stratégies à mettre en place pour relever le défi de la scolarisation primaire universelle des filles.

\subsubsection{Entretiens de groupe}

Les entretiens de groupe ont été réalisés avec les parents d'élèves et les communautés. le but des entretiens de groupe était de susciter les débats entre les parents d'élèves et entre les communautés pour saisir toutes les 
facettes du problème. Dans chacune des localités (Babien, Téguéla et Kani), un entretien de groupe a été réalisé avec les hommes et un autre avec les femmes, soit deux entretiens de groupes par localité. Au total, 6 entretiens de groupe ont été réalisés.

\subsection{Méthode de traitement et d'analyse des données}

Les données ont été traitées à l'aide du logiciel N'vivo. Ce logiciel permet le traitement des données qualitatives. La méthode d'analyse utilisée est l'analyse de contenu. Elle a favorisé l'analyse des rapports d'entretiens individuels et de groupe.

\section{RESULTATS ET DISCUSSION}

\subsection{RESULTATS}

\subsubsection{Obstacles à la scolarisation et au maintien des filles dans l'enseignement primaire}

Les entretiens réalisés avec les communautés et les managers de l'école ont permis d'identifier plusieurs obstacles à la scolarisation et au maintien des filles dans le cycle d'enseignement primaire dans la région du Worodougou.

\section{$\checkmark$ Pauvreté des parents}

Lorsqu'on demande aux populations enquêtées de donner les causes de la non-inscription à l'école et de la déscolarisation des enfants, notamment des filles, elles évoquent en premier lieu les problèmes économiques et financiers. La pauvreté des parents est considérée par les autorités éducatives locales et par les communautés enquêtées comme le premier obstacle à la nonscolarisation et aux déperditions scolaires des filles dans la région du Worodougou. La pauvreté a un impact négatif sur la scolarisation et le maintien des filles à l'école primaire parce que l'éducation a un coût qu'il faut supporter. Son financement n'est pas seulement l'affaire de l'Etat. Les ménages eux aussi contribuent fortement au financement de l'éducation. Ils doivent faire face aux achats de fournitures, aux cotisations du Comité de gestion des établissements scolaires pour l'entretien des écoles, des tenues scolaires et autres dépenses quotidiennes des enfants scolarisés. Or, l'agriculture qui est la principale activité de la région, est selon, les communautés, est caractérisée par un certain nombre de difficultés, notamment les faibles prix d'achat de l'anacarde et du coton. "L'agriculture est l'activité principale des populations vivant dans la région de Séguéla. L'anacarde est la principale culture de rente pratiquée dans la région. Or, selon les populations, les prix d'achat de ce produit sont faibles et ne sont pas respectés par les acheteurs même quand ils sont fixés par le gouvernement. Les pistes d'accès aux champs agricoles n'étant pas de bonne qualité, les populations estiment qu'elles sont obligées soit de brader leur production, soit de la laisser pourrir dans les champs. Le coton est une culture qui pourrait 
permettre aux paysans d'avoir suffisamment de moyens financiers. Mais son prix d'achat est aussi faible. " (Un conseiller pédagogique). Dans ce contexte de pauvreté, de nombreux parents considèrent que les filles peuvent réaliser des activités génératrices de revenus pour soutenir financièrement la famille. Ainsi, elles sont mises en activité économique précoce au détriment de l'école pour ramener des ressources financières dans la famille.

\section{$\checkmark \quad$ Mariages et grossesses précoces}

Pour les personnes interrogées, les mariages et les grossesses précoces observés dans cette région sont un obstacle à la scolarisation et au maintien des filles dans le cycle d'enseignement primaire. A ce sujet, l'un des enquêtés a fait la déclaration suivante : " dans la région du Worodougou, parmi facteurs limitant la scolarisation et la continuité de l'école par les filles, il y a le mariage et les grossesses. Ces facteurs poussent certains parents à refuser de scolariser leurs filles ou les faire sortir leurs filles de l'école primaire, surtout quand elles atteignent l'âge de la puberté" (Conseiller COGES). Les pesanteurs religieuses et culturelles renforcent l'avènement des mariages et des grossesses précoces. Car selon un enseignant interrogé, "les populations de la région qui sont majoritairement analphabètes et musulmanes, estiment que la femme n'a de valeur dans la société que si elle est mariée. De plus, les parents estiment qu'avoir un enfant en dehors du mariage, n'est pas un principe accepté par l'islam. Dès lors qu'un prétendant se signale pour épouser une fille même scolarisée, certains parents n'hésitent pas à la donner en mariage au détriment de la poursuite de l'école ». Par ailleurs, il faut signaler que ce ne sont pas seulement les grossesses au primaire qui favorisent la déscolarisation des filles. Les cas de grossesse précoce dans les établissements d'enseignement secondaire entrainent souvent la déscolarisation de certaines filles de l'école primaire. Certains parents estiment qu'en laissant la fille poursuivre les études jusqu'au collège, la probabilité qu'elle tombe enceinte est grande. Ils préfèrent donc la déscolariser surtout quand elle atteint l'âge de la puberté.

\section{$\checkmark$ Travaux domestiques et champêtres}

Les travaux domestiques et champêtres sont considérés par les acteurs de l'éducation comme un obstacle majeur à la scolarisation et au maintien des filles à l'école primaire. De nos investigations, il ressort que les parents estiment que la maîtrise des travaux domestiques et champêtres par les enfants et notamment par les filles est la base de leur intégration sociale. Par conséquent, les filles qui sont les actrices les plus importantes dans la société doivent les apprendre et les maîtriser. "Les travaux champêtres et domestiques sont considérés comme un apprentissage pour les filles car elles sont appelées à se marier dans un futur proche. Cette situation fait que de nombreuses filles ne sont pas scolarisées et celles qui sont scolarisées sont 
souvent retirées de l'école pendant les heures de cours pour aller au champ ou faire le ménage D'autres filles sont souvent conduites à Abidjan pour s'adonner à des travaux domestiques rémunérés et ce, en pleine année scolaire. Et puis il faut souligner que la plupart des élèves vivent en milieu rural où l'activité principale est l'agriculture. Certaines filles aident leurs mères dans les travaux champêtres et sont privées d'école» (Communauté, Séguéla).

Dans la région, les populations qui sont majoritairement musulmanes accordent une place de choix à la maîtrise des activités domestiques par les filles. Elles considèrent que le principal rôle de la femme est d'effectuer les activités ménagères et que la maitrise des activités domestiques par la population féminine, même celle scolarisée, est donc considérée comme une nécessité. D'une manière générale, les femmes estiment que ces activités sont un apprentissage important pour toute fille qui est appelée à se marier lorsqu'elle sera adulte. Toutefois, il faut relever qu'il est le plus souvent difficile pour certaines filles de concilier ces activités domestiques avec la fréquentation de l'école.

Il est évident l'importance accordée par certains parents aux travaux domestiques au détriment de l'école est une source de sous-scolarisation et de déscolarisation des filles.

\section{$\checkmark$ Ignorance des parents ou persistance de l'analphabétisme}

L'importance du taux d'analphabétisme en Côte d'Ivoire, et principalement dans la région de l'étude est un facteur aggravant du phénomène de sous-scolarisation et de déscolarisation des filles. En effet, le taux d'analphabétisme des adultes en Côte d'Ivoire est estimé à 44,7\% (UNESCO, 2015). En valeur absolue, le nombre d'analphabètes est très élevé. L'analphabétisme est encore plus accentué chez les femmes quelle que soit la tranche d'âge. Selon l'Enquête démographique et de santé et à indicateurs multiples (EDS-MICS) 2011-2012, $61 \%$ des hommes sont considérés comme alphabétisés contre $38 \%$ de femmes. La proportion de femmes analphabètes est plus élevée que celle des hommes (62\% contre $39 \%)$. L'enquête relève que les proportions d'analphabètes diminuent au fil des générations et restent plus élevées parmi les femmes que parmi les hommes : ainsi à 45-49 ans, 47 $\%$ des hommes contre $75 \%$ des femmes sont considérés comme analphabètes ; à 15-19 ans, ces proportions sont respectivement de $32 \%$ et $53 \%$. Le taux de femmes alphabétisées, c'est-à-dire les femmes qui ont, au moins, fréquenté l'école secondaire et celles qui peuvent lire une phrase entière ou une partie de phrase varie de $23,4 \%$ à $43,9 \%$ selon les tranches d'âge (voir tableau cidessous). 
Tableau: Répartition (en \%) des femmes de 15-49 ans par niveau d'instruction atteint et niveau d'alphabétisation et pourcentage de femmes alphabétisées selon les tranches d'âge

\begin{tabular}{|c|c|c|c|c|c|}
\hline GROUPE & NIVEAU & PEUT & PEUT & NE & POURCENTAG \\
S D'AGE & SECONDAIR & LIRE & LIRE & PEUT & E \\
& E OU PLUS & UNE & UNE & PAS & ALPHABETISE \\
& & PHRASE & PARTIE & LIRE & \% \\
& & ENTIER & DE LA & UNE & \\
& & E & $\begin{array}{c}\text { PHRAS } \\
\text { PHRAS } \\
\text { E }\end{array}$ & \\
\hline $\mathbf{1 5 - 2 4}$ & 30 & 10,1 & 3,9 & 55,8 & 43,9 \\
\hline $\mathbf{2 5 - 2 9}$ & 19,8 & 15,2 & 2,9 & 61,9 & 37,8 \\
\hline $\mathbf{3 0 - 3 4}$ & 15,1 & 14,2 & 3,4 & 67,2 & 32,7 \\
\hline $\mathbf{3 5 - 3 9}$ & 14,5 & 15,9 & 2,9 & 66,2 & 33,3 \\
\hline $\mathbf{4 0 - 4 4}$ & 14,8 & 14,5 & 4,7 & 65,3 & 34,0 \\
\hline $\mathbf{4 5 - 4 9}$ & 10,2 & 10,3 & 3,00 & 75,30 & 23,4 \\
\hline
\end{tabular}

Source : Enquête démographique et de santé et à indicateurs multiples (EDS-MICS) 2011-2012

L'analphabétisme des femmes est important dans toutes les régions du pays. Dans la région du Nord-Ouest, région de l'étude, $5,1 \%$ des femmes âgées de 15 à 49 ans ont un niveau d'étude secondaire ou plus, 5\% d'entre elles sont capables de lire une phrase entière, $1,6 \%$ des femmes peuvent lire une partie d'une phrase et $88,1 \%$ ne peuvent pas lire une phrase. Dans cette région, le taux de femmes alphabétisées est de 11,7\% (tableau ci-dessous).

Tableau : Répartition (en \%) des femmes de 15-49 ans par niveau d'instruction atteint et niveau d'alphabétisation et pourcentage de femmes alphabétisées selon les régions.

\begin{tabular}{|c|c|c|c|c|c|}
\hline REGION & $\begin{array}{c}\text { NIVEAU } \\
\text { SECONDAI } \\
\text { E OU PLUS }\end{array}$ & $\begin{array}{c}\text { PEUT } \\
\text { LIRE } \\
\text { UNE } \\
\text { PHRASE } \\
\text { ENTIER } \\
\text { E }\end{array}$ & $\begin{array}{c}\text { PEUT } \\
\text { LIRE } \\
\text { UNE } \\
\text { PARTIE } \\
\text { DE LA } \\
\text { PHRAS } \\
\text { E }\end{array}$ & $\begin{array}{c}\text { NE } \\
\text { PEUT } \\
\text { PAS } \\
\text { LIRE } \\
\text { UNE } \\
\text { PHRAS } \\
\text { E }\end{array}$ & $\begin{array}{c}\text { POURCENTAG } \\
\text { E } \\
\text { ALPHABETISE } \\
\%\end{array}$ \\
\hline $\begin{array}{c}\text { Centre } \\
\text { Centre- } \\
\text { Est }\end{array}$ & 21,6 & 11,5 & 5,1 & 61,7 & 38,2 \\
\hline $\begin{array}{c}\text { Centre- } \\
\text { Nord }\end{array}$ & 18,7 & 12,1 & 6,6 & 57,3 & 42,4 \\
\hline $\begin{array}{c}\text { Centre- } \\
\text { Ouest }\end{array}$ & 16,8 & 10,5 & 4,7 & 66,3 & 33,7 \\
\hline Nord & 12,8 & 10,9 & 2 & 70,3 & 29,7 \\
\hline Nord-Est & 14,5 & 9,6 & 4,2 & 71,4 & 28,2 \\
\hline
\end{tabular}




\begin{tabular}{|c|c|c|c|c|c|}
\hline $\begin{array}{c}\text { Nord- } \\
\text { Ouest }\end{array}$ & 5,1 & 5 & 1,6 & 88,1 & 11,7 \\
\hline Ouest & 12,1 & 12,3 & 1,4 & 72,6 & 25,8 \\
\hline Sud & 21,4 & 14,6 & 2,2 & 61,8 & 38,2 \\
\hline $\begin{array}{c}\text { Sud- } \\
\text { Ouest }\end{array}$ & 13,3 & 15,6 & 2,2 & 68,4 & 31,2 \\
\hline $\begin{array}{c}\text { Ville } \\
\text { d'Abidja } \\
\text { n }\end{array}$ & 37,3 & 16,2 & 6,1 & 40 & 59,6 \\
\hline
\end{tabular}

Source : Enquête démographique et de santé et à indicateurs multiples (EDS-MICS) 2011-2012

L'analyse des tableaux précédents montre que le phénomène d'analphabétisme reste un défi à relever en Côte d'Ivoire, chez les femmes et surtout dans certaines régions y compris celle de l'étude. Cette situation entraine des difficultés majeures au niveau de la scolarisation, mais aussi du maintien des filles dans l'enseignement primaire. «La plupart des parents qui refusent de scolariser leurs filles sont le plus souvent des gens qui n'ont jamais été scolarisés. Les filles aussi qui sont déscolarisées ont des parents analphabètes. Cela montre que de nombreux parents analphabètes ne comprennent pas encore le bienfondé de l'école et plus précisément la scolarisation des filles. " (Inspecteur de l'enseignement préscolaire et primaire).

Les filles scolarisées dont les parents sont analphabètes sont victimes le plus souvent de l'absence de suivi et d'encadrement. Cette absence de suivi et d'encadrement peut engendrer le redoublement répété qui est considéré comme une cause d'abandon scolaire par les filles. De nombreux parents analphabètes continuent de négliger la scolarisation et l'encadrement de leurs filles parce qu'ils estiment qu'elles peuvent se marier en cas d'échec scolaire et ne sont pas, par conséquent obligées d'aller à l'école. Cette situation, malgré l'obligation de l'école primaire votée au parlement, est obstruant pour la réalisation de la scolarisation primaire universelle des filles. "Etant donné qu'elles sont appelées à quitter la famille pour se marier, les filles sont négligées et ne sont pas bien suivies par les parents. Lorsque, dans une même famille, des filles et des garçons sont scolarisés, les parents mettent l'accent sur l'encadrement et le suivi des garçons au détriment des filles" (Communauté, Séguéla).

\section{$\checkmark$ Emigration interne (vers Abidjan) et externe (vers l'occident)}

L'existence d'une émigration interne et externe des jeunes filles est considérée comme un obstacle majeur à la scolarisation des filles et un facteur favorisant de leur déscolarisation. En effet, il ressort des entretiens avec les différents 
acteurs que de nombreuses filles d'âge scolaire, soutenues le plus souvent par leurs parents et principalement par leurs mères, rêvent de quitter leur région pour d'autres destinations avec l'espoir d'y améliorer leur bien-être et partant celui de la famille. Les destinations prisées sont l'occident, plus précisément la France (immigration externe) et le cas échéant, Abidjan, la capitale économique (immigration interne) qui concentre la plupart des activités industrielles et économiques de la Côte d'Ivoire. Cette émigration est plus accentuée dans le département de Kani. La particularité de cet exode, selon l'Inspecteur de l'Enseignement Préscolaire et Primaire, est qu'il est pratiqué essentiellement par les femmes et les filles. "Les femmes originaires de la région du Worodougou, vivant en Europe et principalement en France ont construit de belles maison dans la ville de Kani. Et qu'elles viennent en vacances, elles ont de belles voiture. Cette situation fait que chaque famille veut avoir une fille en Europe, notamment en France. Dans ce contexte, de nombreuses filles ne sont pas scolarisées parce que leurs parents ambitionnent de les faire partir en Europe. Cette situation aussi fait que certaines filles scolarisées sont encouragées par leurs mères à abandonner les études pour tenter l'aventure européenne, car les femmes de la région qui vivent en Europe, bien qu'elles soient analphabètes, sont enviées et considérées comme des modèles à imiter. "

En clair, la plupart des femmes qui ont réussi ne l'ont pas été grâce à l'école. Elles ont réussi parce qu'elles sont allées en Europe. Le phénomène, d'exode, constitue donc une source de démotivation pour les filles scolarisées qui rêvent de partir chercher le bien être socioéconomique dans ces pays du nord.

\section{$\checkmark$ Représentation sociale de la femme dans les communautés}

Les représentations sociales de la femme dans les communautés sont un facteur explicatif de la non-scolarisation des filles et de leur non-maintien à l'école primaire. En effet, les comportements et les attitudes réfractaires que les parents ont de la scolarisation et du maintien de la fille à l'école sont fortement influencés par les représentations sociales que les communautés ont de la femme.

Quand on demande aux communautés de donner les rôles principaux d'une femme dans la société, les mots ou expressions qui reviennent le plus souvent sont : ménagère, procréatrice, personne subordonnée à l'homme, personne dépendante de l'homme, éducatrice des enfants, une personne courageuse qui prend soin de la famille, une personne devant pratiquer les travaux champêtres, sexe faible, objet sexuel ou encore être inférieur aux hommes.

Cette image qu'ont les communautés de la femme, n'est pas de nature à favoriser la scolarisation et la rétention des filles à l'école primaire. 


\section{$\checkmark$ Représentation sociale de l'école par les communautés de la région}

Certains parents analphabètes ou peu scolarisés ont une certaine représentation sociale de l'école qui n'est pas de nature à favoriser la scolarisation primaire, plus précisément celle des filles. Ceux là estiment que la finalité l'école, c'est l'acquisition d'un emploi rémunéré et par conséquent avoir suffisamment l'argent. Or, on peut avoir l'argent sans avoir été à l'école, en faisant d'autres activités. Partant de cette perception qu'elles ont de l'école, ces personnes sont réfractaires à la scolarisation des enfants et surtout la scolarisation des filles. Le nombre d'années de fréquentation scolaire est donc vu comme une perte de temps. " Nos parents estiment que l'objectif de l'école est de permettre aux enfants d'avoir plus tard un travail bien payé. Or, le nombre d'années qu'un enfant passe à l'école avant d'avoir d'être diplômé et d'avoir un emploi est considéré comme une perte de temps parce que l'enfant peut apprendre un métier ou quand il s'agit des filles, les parents pensent qu'elles peuvent faire du commerce pour avoir de l'argent ou se marier plus tard avec quelqu'un. " (Conseiller COGES). Cette thèse est soutenue par certaines femmes analphabètes qui estiment que les filles peuvent pratiquer des activités génératrices de revenu au lieu de perdre le temps dans les écoles où il n'est pas évident qu'à la fin qu'elles auront du travail.

\section{$\checkmark$ Rareté des femmes dans le Worodougou ayant réussi grâce à l'école}

La rareté de modèles féminins sortis des écoles primaires de la région et ayant réussi grâce à l'école est considérée comme un obstacle à la scolarisation et au maintien des filles à l'école primaire. Cette rareté de modèles féminin constitue une source de démotivation chez certains parents qui ne veulent pas scolariser leurs filles, mais aussi une source de démotivation chez les filles scolarisées qui peuvent à un moment donné abandonner la fréquentation de l'école. En effet, dans la région de Séguéla et principalement en milieu rural, beaucoup d'écoles ont été construites dans les années 1970. Cependant, les cas de réussites en ce qui concerne les filles sont rares. Le fait qu'il n'y ait pas de filles sorties de ces écoles et ayant réussi grâce à l'école peut être une source de démotivation aussi bien pour les filles elles-mêmes que pour les parents analphabètes. Prenant 1'exemple des Ecoles primaires publiques de Wongué et de Babien, un conseiller pédagogique nous a relaté ceci : " l'Ecole primaire publique de Wongué cette école a été construite en 1976. Depuis cette époque jusqu'à aujourd'hui, aucune fille n'a encore réussi grâce à l'école, le seul cadre du village, a-t-il affirmé, est un infirmier. A l'EPP Babien, la seule fille ayant réussi grâce à l'école est une aide soignante dans un village voisin. Pourtant, l'école a été construite au milieu des années 1970. »

La rareté des modèles féminins ayant fréquenté les écoles de la région constitue un obstacle sérieux à la scolarisation primaire universelle des filles, 
parce que certains parents finissent par croire que l'école n'est pas faite pour les filles.

\section{$\checkmark$ Discontinuité éducative}

De manière générale, l'offre d'éducation primaire n'est pas suffisante et pose quelques fois des difficultés relatives à la scolarisation et au maintien des enfants et notamment des filles à l'école. En effet, lorsque, dans une école, il se pose le problème de l'insuffisance de places, les filles sont sacrifiées au profit des garçons. Au cours des entretiens, il est ressorti que les populations locales préfèrent scolariser les garçons en cas de manque de place. On note aussi que les écoles comprenant seulement trois du primaire (CP1, CP2 et CE1) sont un facteur favorisant de l'abandon scolaire.

Au niveau national, $75 \%$ des écoles publiques proposent les six niveaux de l'enseignement primaire en 2013/2014. Dans ce contexte, ce sont $11 \%$ des enfants (RESEN, 2015) qui potentiellement sont à risque d'abandonner l'école suite à la discontinuité éducative.

\subsubsection{Actions passées et actuelles en faveur de la scolarisation et du maintien des filles à l'école}

Les stratégies passées et actuelles se situent au niveaux central, déconcentré et local.

Au niveau central, on note la décision du gouvernement depuis une décennie de rendre l'école gratuite. Dans la région du Worodougou, certains enfants bénéficient d'ouvrages scolaires gratuits. Toutefois, il faut relever que la grande partie des élèves dont de nombreuses filles ne bénéficient pas de cette gratuité. "L 'école gratuite initiée par le gouvernement est plus un slogan qu'une réalité. D'abord, il faut noter que les livres fournis ne viennent pas toujours à temps; ensuite, il faut relever que, compte tenu de l'augmentation du nombre d'enfants à scolariser, les fournitures du gouvernement ne suffisent pas du tout, parce que la majorité des élèves y compris les filles, $n$ 'en profitent pas. Enfin, il faut dire que des cotisations sont faites régulièrement par les élèves dans le cadre du COGES. Si vous n'êtes pas à jour de vos cotisations, les enseignants chassent vos enfants de l'école. " (Leader communautaire).

Au niveau de la région de Séguéla, il faut dire que les actions en faveur de la scolarisation et du maintien scolaire des filles dans l'enseignement primaire sont rares et se résument en des campagnes de sensibilisation. Toutefois, cette rareté des actions pourrait aussi s'expliquer par l'absence d'ONG dans la région intervenant dans le domaine de l'éducation. En effet, seule l'ONG le Worodougou Espoir intervient dans le domaine. Elle met cependant un accent plus particulier sur l'alphabétisation des adultes. Les campagnes de sensibilisation dans la DREN de Séguéla consistent en général, en l'organisation de réunions avec les parents d'élèves pour leur expliquer le bien-fondé de la scolarisation et du maintien des filles dans le cycle 
d'enseignement primaire. "On a organisé des campagnes de sensibilisation des parents d'élèves; et puis, nous encourageons et motivons les filles en leur offrant des cadeaux " (IEEP, Séguéla). D'autres acteurs font la sensibilisation à travers des sketches. "On sensibilise les parents d'élèves de l'IEEP, les chefs de communautés, les autorités politiques et administratives. Cette sensibilisation se fait souvent à travers des sketches " (IEEP, Séguéla). Ces campagnes sont généralement organisées par les chefs d'établissement, les responsables de l'IEEP et du COGES. Toutefois, l'UNICEF aurait apporté un soutien scolaire pour que le la scolarisation et le maintien des filles soit une réalité dans la région. "Il y a eu des campagnes de sensibilisation organisées par la direction de l'école et l'UNICEF, qui à cette occasion, avait remis des cartables aux filles " (Communauté, Séguéla)

\subsubsection{Stratégies pour relever le défi de la scolarisation primaire universelle des filles}

La réalisation de l'école primaire universelle implique la mise en œuvre effective de plusieurs stratégies à plusieurs niveaux et par plusieurs acteurs. D'une manière générale, ces stratégies consisteront à amener et à maintenir les filles à l'école primaire, à réduire les écarts entre de scolarisation entre les filles et les garçons tout en garantissant un enseignement et un apprentissage de qualité.

\section{$\checkmark$ Au niveau de l'Etat}

L'Etat devra élaborer une politique cohérente et réalisable en matière de scolarisation et de maintien des filles jusqu'à l'âge de 16 ans conformément à la loi sur l'école obligatoire votée par le parlement en septembre 2015. Cette politique devra mettre un accent particulier sur certains aspects importants.

\section{- Rendre effective la gratuité totale de l'école}

L'effectivité de la gratuité de l'école combinée avec son obligation conduira à des écoles satisfaisantes en matière de scolarisation et de maintien des filles à l'école primaire. Dans cette perspective, le gouvernement doit penser à une prise en charge intégrale des filles à scolariser et scolarisées en mettant à leur disposition des kits scolaires et vestimentaires complets et à temps. Cette stratégie serait une réponse appropriée au problème de pauvreté des parents évoqué comme obstacle à la scolarisation et au maintien des filles à l'école.

\section{- Accroitre l'offre de l'éducation}

Une augmentation du nombre des établissements primaire avec les six niveaux requis conduirait à une réduction de la discontinuité éducative chez les filles. "Lorsque les filles doivent quitter une école où elles fréquentaient pour aller poursuivre dans une autre école, les parents, notamment les mères n'hésitent pas à les faire sortir de l'école. Il est donc nécessaire que toutes les 
écoles primaires au niveau national et au niveau de la région soient complètes avec les six niveaux d'enseignement pour éviter que des enfants se retrouvent en dehors de l'école " (Conseiller COGES). L'accroissement de l'offre de l'éducation devra aussi concerner le recrutement en nombre suffisant d'enseignants bien formés. Augmenter aussi le nombre de femmes dans le corps enseignant ainsi qu'au niveau des directions d'établissements est une stratégie qui favoriserait la scolarisation et le maintien des filles à l'école primaire.

\section{- Mettre en œuvre des programmes d'alphabétisation}

Relever le défi de la scolarisation primaire universelle dans un délai raisonnable nécessite la mise en œuvre des projets ou programmes d'alphabétisation des adultes, notamment en faveur des femmes. L'analphabétisme des adultes, et surtout celui des femmes étant identifié comme un obstacle majeur à la scolarisation et au maintien des filles à l'école primaire, il est primordial que les autorités éducatives songent à mettre en œuvre des activités d'alphabétisation dans la région. L'ouverture de centres d'alphabétisation pour les femmes aussi bien en milieu urbain qu'en milieu rural contribuerait à augmenter non seulement les taux d'inscription des filles et leur à l'école, car les femmes alphabétisées sont plus disposées à scolariser leurs filles que celles qui ne le sont pas. "Dans les villages où l'on a initié des activités d'alphabétisation des femmes, on a constaté que le taux de scolarisation des filles a augmenté, et la déscolarisation est en baisse. Il faut donc que des projets d'alphabétisation soient initiés dans toute la région pour les adultes et principalement pour les femmes. » (Enseignant, Kani).

\section{- Accroitre le nombre de cantines scolaires}

Les cantines scolaires font partie des stratégies de maintien des filles dans le cycle primaire. Leur existence dans l'établissement est une source de motivation pour les filles de poursuivre les études jusqu'à la classe de CM2. "Les cantines scolaires jouent un rôle important dans la scolarisation des filles et leur maintien à l'école. En général, lorsqu'il n'y a pas de cantine, ce sont les filles elles-mêmes qui font la cuisine à midi pour leur déjeuner avant de revenir à l'école dans l'après. Or, avec la présence d'une cantine, elle ne fait pas de cuisine à midi et elle mange très bien. » (IEEP Séguéla). Au niveau national, seules $35 \%$ des établissements d'enseignement primaire ont une cantine fonctionnelle. Dans la région de Séguéla, $43 \%$ des écoles primaires ont une cantine. Ce pourcentage est certes au dessus de la moyenne nationale, mais il faut relever que Séguéla étant une région de sous-scolarisation et de déscolarisation des filles, il importe d'accroitre davantage le nombre de cantines scolaires pour stimuler la demande d'éducation primaire, notamment chez les filles. 


\section{$\checkmark$ Au niveau de l'école, des organisations internationales d'éducation et des communautés}

Etablir une franche collaboration entre les différents acteurs de l'école et les communautés locales est un moyen pouvant conduire à une amélioration du taux de scolarisation des filles. Cette collaboration entre parents d'élèves et enseignants permettra de réaliser la sensibilisation et la motivation des filles. Les leaders communautaires devront occuper une place important dans cette collaboration dont le but essentiel sera d'amener les communautés à mieux comprendre la nécessité de la scolarisation et du maintien de la fille à l'école primaire.

\section{$\checkmark$ Au niveau de la famille et de l'élève}

Réaliser des vastes campagnes de communication, d'information et de sensibilisation sur la nécessité de scolariser les filles et de les laisser poursuivre les études.

A l'endroit des parents d'élèves, la sensibilisation mettra l'accent sur le renforcement de l'encadrement moral et scolaire des filles scolarisée et sur l'importance d'éviter les mariages précoces des filles qui empêchent la scolarisation ou qui débouchent naturellement sur des grossesses qui mettent un terme à la fréquentation de l'école des filles. Il faudra aussi faire la sensibilisation à la réduction du temps de travail domestique au profit de l'école des filles.

A l'endroit des filles scolarisées, la sensibilisation portera sur la prise de conscience réelle de l'intérêt d'aller à l'école et des comportements à risque et à la prise de conscience que la réussite scolaire passe par la réalisation des efforts personnels pour l'apprentissage scolaire. La sensibilisation devra prouver aux jeunes filles que la réussite scolaire n'est pas sexuée, et qu'elles peuvent réussir comme les garçons. De ce fait, les campagnes de sensibilisation devront prendre des exemples de femmes connues au niveau national, qui ont fait de longues études et qui occupent des postes de responsabilité. Pour que ces campagnes de sensibilisation puissent produire des résultats satisfaisants, il faut impliquer un ensemble d'acteurs : les leaders communautaires (chefs religieux, chefs de villages, associations des femmes, les filles, les médias nationaux et locaux, etc.), les structures déconcentrées de l'éducation nationale, les élus locaux et des responsables de la diaspora.

\subsection{DISCUSSION DES RESULTATS}

Le problème de la scolarisation primaire prôné par les dirigeants africains aux lendemains de l'accession à l'indépendance demeure, plus de 40 ans après, une préoccupation majeure pour la plupart des pays au sud du Sahara. De nombreux pays du continent, caractérisés par des faibles taux de scolarisation primaire, par des taux de déscolarisation et d'abandon scolaire 
importants, n'ont pu atteindre les Objectifs de l'Education Pour Tous dont celui de l'enseignement primaire universel. La Côte d'Ivoire qui fait partie de ces pays, a certes progressé en matière de scolarisation, mais des obstacles subsistent surtout en ce qui concerne l'éducation primaire universel, et principalement celui des filles. Pour favoriser la scolarisation primaire universelle et surtout celle des filles, le parlement ivoirien a adopté une loi en septembre 2015 pour rendre l'école obligatoire jusqu'à l'âge de 16 ans. Pour Bah (2015), la loi sur l'école obligatoire est une réponse à la scolarisation des filles car tous les enfants doivent aller à l'école de 6 à 16 ans. Pour nous, cette seule loi ne suffit pas pour résoudre les problèmes de sous-scolarisation et de déscolarisation des filles. Il est important d'identifier les obstacles à ces problèmes et chercher à apporter des réponses appropriées qui passent par la mise en œuvre concrète de stratégies que nous avons identifiées au terme de cette investigation. Pour rappel, ces stratégies concernent l'augmentation de l'offre de l'éducation primaire, la mise en œuvre de projets ou de programmes d'alphabétisation en faveur des adultes analphabètes, et principalement en faveur des femmes, l'effectivité de l'école gratuite pour réduire les effets de la pauvreté sur la scolarisation des filles, la création de cantines scolaires dans toutes les écoles primaires, la franche collaboration entre les acteurs de l'éducation et surtout l'élaboration d'une politique de communication, d'information et de sensibilisation sur la nécessité de la scolarisation et du maintien des filles à l'école. Le Rapport d'Etat du Système Educatif National 2015 montre que la non-déclaration des naissances à l'état civil constitue un obstacle à la scolarisation primaire universelle inclusive. Pour lever cet obstacle (Plan National de Développement 2016-2020), le gouvernement ivoirien veut veiller à l'enregistrement de toutes les naissances et des enfants de moins de 15 ans non enregistrés à l'état civil, filles comme garçons, afin de faciliter leur scolarisation. Ce Plan annonce même d'autres stratégies envisagées par le gouvernement ivoirien afin de réaliser la scolarisation primaire universelle, et principalement celle des filles. Il s'agit entre autres de la réhabilitation de plus 1400 salles de classes, de la construction de plus de 10.000 nouvelles salles de classes, du recrutement de plus de 18.000 enseignants du primaire, de l'augmentation des rationnaires et de la distribution gratuite des manuels et de kits scolaires qui demeure encore un rêve pour de nombreux parents d'élèves malgré la gratuité de l'école annoncée depuis l'année 2000. Autre stratégie annoncée, c'est la réalisation d'un Programme National d'Education pour Tous avec la promotion de la scolarisation et du maintien durable des filles à l'école. Cependant, les modalités de mis en œuvre de ce programme ne sont pas encore explicitées.

Le Ministère de la Solidarité, de la Famille, de la Femme et de l'Enfant (2014) propose un accroissement de l'offre d'éducation, une mobilisation des collectivités locales et surtout des femmes en vue d'appuyer l'éducation des 
filles dans leurs circonscriptions et localités et l'élaboration de plans de promotion des filles. Comme on le constate, les recherches sur la question de la scolarisation primaire universelle des filles ont permis d'identifier des obstacles et de voir les stratégies à mettre en œuvre pour atteindre l'objectif visé. Cependant, il faut relever que ces recherches ne donnent pas des stratégies spécifiques aux zones régions caractérisées par la sous-scolarisation et la déscolarisation des filles, comme c'est le cas de la région du Worodougou dans le nord-ouest de la Côte d'Ivoire. dans cette région, l'un des obstacles majeurs à la scolarisation et au maintien des filles à l'école primaire, que des recherches à notre avis, n'ont pas encore identifié, est l'émigration des femmes et des filles de cette région vers l'occident, et plus précisément vers la France.

Cette émigration fait rêver de nombreuses jeunes filles d'âge de la région qui ne pensent plus à aller à l'école, mais plutôt à partir en France. C'est pourquoi, nous estimons que dans les stratégies à mettre en œuvre dans le Worodougou, les femmes et les hommes de la diaspora originaire de cette région devront jouer un rôle essentiel.

\section{Conclusion}

Aux lendemains des indépendances, de nombreux pays africains ambitionnaient de réaliser en très peu d'années, la scolarisation primaire universelle. Depuis, la Conférence des Ministres de l'Education tenue à Addis Abeba en Ethiopie en 1961 jusqu'au Forum mondial sur l'éducation qui s'est déroulée à Dakar et l'Assemblée de Nations Unies sur les Objectifs du Millénaire pour le Développement en 2000, en passant par la conférence de Jomtien en 1990 sur l'Education Pour Tous, les dirigeants africains ont toujours pris l'engagement de réaliser la scolarisation primaire inclusive.

Malgré tous les engagements aux niveaux mondial, africain et national, la sous-scolarisation et la déscolarisation, notamment en ce qui concerne les filles demeurent une préoccupation pour de nombreux pays dont ceux du contient africain. Les autorités politiques ivoiriennes ambitionnaient, après l'indépendance du pays, de réaliser la scolarisation primaire universelle inclusive en une vingtaine d'années. Ce défi n'était cependant pas facile à relever parce que, bien avant l'indépendance, la politique coloniale n'avait pas tablé sur une scolarisation primaire massive. Aujourd'hui, la nécessité du développement humain durable impose aux autorités ivoiriennes l'adoption d'une politique d'éducation inclusive. Il urge donc de mettre en œuvre des stratégies appropriées en vue de la scolarisation primaire universelle des filles partout en Côte d'Ivoire et principalement dans les régions a faible taux de scolarisation et à fort taux de déscolarisation dont la région du Worodougou.

Toutefois, l'obligation de l'école adoptée par le parlement soulève des réflexions. Quelles sont les moyens dont dispose le gouvernement pour contraindre les populations à scolariser leurs enfants? 


\section{References:}

Bah, B. (2015). Scolarisation des filles : pour avancer lever les obstacles dans le monde et même en France; Communication au cours de la 5ème quinzaine de l'égalité femmes-hommes de la Région Rhône-Alpes ; Lyon.

BIH, E (2003), Etude sur l'égalité des sexes dans le domaine de l'éducation en Côte d'Ivoire, Abidjan, ROCARE Côte d'Ivoire.

DEDY S. et BIH E., (1997). Etude des déterminants familiaux de la scolarisation des filles et des enfants en zone de sous scolarisation de Côte d'Ivoire, rapport final, Abidjan, MENFB/DPES /ROCARE Côte d'Ivoire.

FORUM DES EDUCATRICES AFRICAINES (2010). Renforcer la recherche sur le genre pour améliorer l'éducation des filles et des femmes en Afrique. Les cahiers de recherche du FAWE ; Volume 1/2010 ; 156 pages.

Illou A (2011). La scolarisation des filles au Niger : obstacles socioculturels, reflets actions sud, numéro 2, décembre 2011; disponible sur le site www.reflets-actions-sud

Lange M.F. (1998) (dir), L'école et les filles en Afrique. Scolarisation sous conditions, Paris, Karthala.

Manikak, O. (2011). L'achèvement des études des filles au Cameroun : freins culturels et psychologiques; numéro 2, décembre; disponible sur le site www.reflets-actions-sud

MENET, MESRS, METFP (2010). Plan d'actions à moyen terme du secteur de l'éducation, 2010-2013, Abidjan, 106 pages.

MENET, MESRS, METFP (2015). Rapport d'Etat du Système Education National, Abidjan.

Ministère de l'Education Nationale et de l'Enseignements Technique (2014). Rapport d'analyse statistique; Abidjan, DPES ; 65 pages.

Ministère de l'Education Nationale et de l'Enseignements Technique (2014). Examen national 2015 de l'Éducation pour tous : Côte d'Ivoire". Rapportbilan de mise en ouvre de l'EPT en Cote d'Ivoire 2000/2014 ; 29 pages.

Ministère de la Solidarité, de la Famille, de la Femme et de l'Enfant (2014). Rapport de la Côte d'Ivoire sur la mise en ouvre du Programme d'Action de Beijing vingt ans après, Abidjan, 55 pages.

Ministère d'Etat, Ministère du Plan et du Développement, INS. (2006). Enquête par graphes à indicateurs multiples ; 199 pages.

Ministère du Plan et du Développement (2016). Plan national de développement 2016-2020 : Diagnostic stratégique de la Côte d'Ivoire sur la trajectoire de l'émergence, Tome 1, Abidjan, MPD

Ministère du Plan et du Développement (2016). Plan national de développement 2016-2020: Orientations stratégiques, Tome 2, Abidjan, MPD.

ONU (2015). Rapport sur les Objectifs du Millénaire pour le Développement ; New York ; 72 pages 
PNUD (2012): Les échos du PNUD, Bulletin d'information du PNUD Côte d'Ivoire, $\mathrm{N}^{\circ} 2$.

PNUD, Union Africaine, Bad, Commission Economique pour l'Afrique (2012) : Rapport OMD 2012. Évaluation des progrès accomplis en Afrique dans la réalisation des objectifs du Millénaire pour le développement ; 186 pages

UNESCO (2014). Rapport mondial de Suivi de l'Education Pour Tous 2013/2014 ; Paris, UNESCO ; 444 pages

UNESCO (2015). Rapport mondial de Suivi de l'Education Pour Tous 2015 ; Paris, UNESCO ; 453 pages

UNICEF (2012). Situation des enfants dans le monde, téléchargeable sur le site

http://www.unicef.org/french/sowc2012/pdfs/SOWC\%202012\%20Main\%20 Report\%20LoRes\%20PDF_FR_03132012.pdf 